Plenary 1:

Enhancing inclusivity and diversity into the new norm: what can we do better in medical education?

\title{
Evolving trends for assessment in the new norm of medical education
}

\begin{abstract}
Eraky, M.A.
Saudi Arabia

Many scholars used to advocate 'change' in medical education, but almost nobody anticipated the fast and furious transformation of assessment practices over a period of months, rather than years. We need to reflect on lessons learned and explore new frontiers in assessment in the new norm of medical education.

Apart from the application of technology, perhaps we need to start by asking more fundamental questions, such as: Do we need to revisit the purpose(s) of assessment? How far are we ready to trust subjective inputs, not only from experts, but from trained observers at the workplace? How does the local context regulate assessment of professionalism? Why our A+graduates are not necessarily the most successful ones in real practice? How to improve the predictive validity of our assessment tools? And finally, how far we are willing to pay more attention to assess implicit attributes that have a great impact on the future practice of graduates?
\end{abstract}

